\title{
INCREMENTO DE ÁREAS IMPERMEABLES POR CAMBIOS DE USOS DE LA TIERRA EN LA MICROCUENCA DEL RÍO BURÍO
}

\author{
Ramón Masís Campos ${ }^{1 *}$ \\ ramon.masiscampos@ucr.ac.cr \\ Hubert Vargas Picado ${ }^{2}$ \\ hvp.ucr@gmail.com
}

\begin{abstract}
Resumen
La investigación se realizó en la microcuenca del río Burío, Heredia, Costa Rica. Se analizaron tres escenarios de impermeabilización de suelos por cambios de uso de la tierra en los años 1960, 1990 y 2010. Los resultados demostraron una sustitución de coberturas agroforestales por urbanas; es decir, impermeables. El conjunto de superficies impermeables han alterado la respuesta hidrológica del área de estudio, respecto a la capacidad de la superficie de favorecer o dificultar el escurrimiento directo, la infiltración o la interceptación del agua. Esto ha repercutido en aumento de caudales pico en una microcuenca constituida por espacios naturales o poco alterados.

Palabras clave: Cambios de uso de la tierra, escenarios de impermeabilización de suelos, microcuenca, rio Burío.
\end{abstract}

\begin{abstract}
Three scenarios were analyzed to show by changes of land use in the years 1960, 1990 and 2010.The results demonstrated a replacement rural covers for urban development. The impervious surfaces have altered the hydrological response watershed for the ability of the surface to promote or hinder direct runoff, infiltration of water or interception. This has resulted in increased peak flows in a watershed.

Key words: Changes, landuse, impervious surfaces, watershed, Burío river.
\end{abstract}

\section{Introducción}

En una cuenca hidrográfica, el cambio del uso de la tierra de condiciones rurales a urbanas altera la respuesta hidrológica, especialmente el escurrimiento de las aguas, ya que en varios tramos de los ríos, estos no son capaces de transportar el excedente de escorrentía potenciado por la impermeabilización de suelos. Superficies como cementos y asfaltos disminuyen la capacidad de infiltración y limita a las coberturas vegetales para interceptar la lluvia. Esto altera el régimen de descarga de la cuenca ante una tormenta intensa de corta duración, y produce inundaciones con alto poder erosivo y transporte de materiales aguas abajo.

En Costa Rica, es evidente el cambio del uso de la tierra a través del tiempo, especialmente en la Gran Área Metropolitana (GAM). Para el caso de las microcuencas de la GAM, la exposición de las muchas comunidades aumenta debido al crecimiento del espacio urbanizado en las

1, 2 Escuela de Geografía, Sede Rodrigo Facio, Universidad de Costa Rica.

Este artículo forma parte del trabajo final de graduación: "Análisis de los cambios del uso de la tierra en 1960, 1990, 2010 y su efecto en el comportamiento hidrológico en la microcuenca del río Burío, Heredia, Costa Rica". 
últimas décadas, lo cual provoca la alteración de la cobertura original de las cuencas hidrográficas y la dinámica fluvial.

Este predominio del uso urbano en la GAM ha modificado los patrones de drenaje y ha aumentado la escorrentía superficial, por efecto de la impermeabilización de los suelos con asfalto, zinc, canoas, cemento, alcantarillado, entre otros (MIVAH, PNUMA y Observatorio del Desarrollo, 2006). Producto de esto, las poblaciones ubicadas en las partes bajas de las microcuencas son impactadas por inundaciones cada vez más frecuentes.

Stanuikynas y Van (2000:1) definen el concepto de impermeabilización de suelos como "aquellas superficies que evitan el movimiento del agua hacia el interior del suelo. Las causas suelen ser naturales (geológicas), antrópicas (compactación de los suelos) o una combinación de estas; además, el grado de impermeabilización varía con relación a los materiales que conforman la superficie impermeabilizante y a procesos físicos y químicos que actúan en estos".

Las perturbaciones hidrológicas provocados por la impermeabilización de suelo han sido estudiadas por Ruberto, Deprettis, Pilar, Prieto, Gabazza y Zarate (2006), y ellos han concluido tres impactos relevantes.

1. Reducción del promedio anual de evapotranspiración y consecuentes cambios en la cantidad de agua que circula en la cuenca, en el tiempo y volumen de las precipitaciones, en tiempos y tasas de carga y descarga.
2. Disminución del tiempo de retardo de la cuenca, debido a que el agua circula por superficies menos rugosas y adquiere mayor velocidad de escurrimiento.

3. La cuenca se torna más sensible ante tormentas intensas de corta duración.

Como se ha justificado, la impermeabilización progresiva de una microcuenca, principalmente en su parte alta y media, es un factor primordial en las descargas torrenciales en las áreas con pendientes más suaves.

Para el caso de la microcuenca del río Burío, el efecto negativo de la impermeabilización de suelos se ha manifestado con avenidas torrenciales en las últimas décadas, principalmente en el cantón de Belén, donde se han reportado inundaciones en 1981, 2004, 2007 y 2008; esto ha afectado a la población residente, principalmente con daños en viviendas y puentes.

\section{Área de estudio}

La microcuenca del río Burío drena un área aproximada de $22 \mathrm{~km}^{2}$ y forma parte de la subcuenca del río Virilla. Esta área forma parte de los cantones de San Rafael, Barva, Heredia, Flores, Belén y el distrito San Rafael de Alajuela.

Sus principales cursos de agua son quebrada Seca, quebrada Aries y río Burío, los cuales recorren aproximadamente $19 \mathrm{~km}$ desde la naciente cercana al poblado de Getsemaní (San Rafael de Heredia) hasta la bifurcación con el río Bermúdez (San Rafael de Alajuela). Su localización geográfica está definida por las coordenadas medias $10^{\circ} \mathrm{N}$ y $84^{\circ} 9^{\prime} \mathrm{O}$ (Figura 1 ). 
Figura 1

Área de estudio: microcuenca del río Burío, Heredia, Costa Rica.

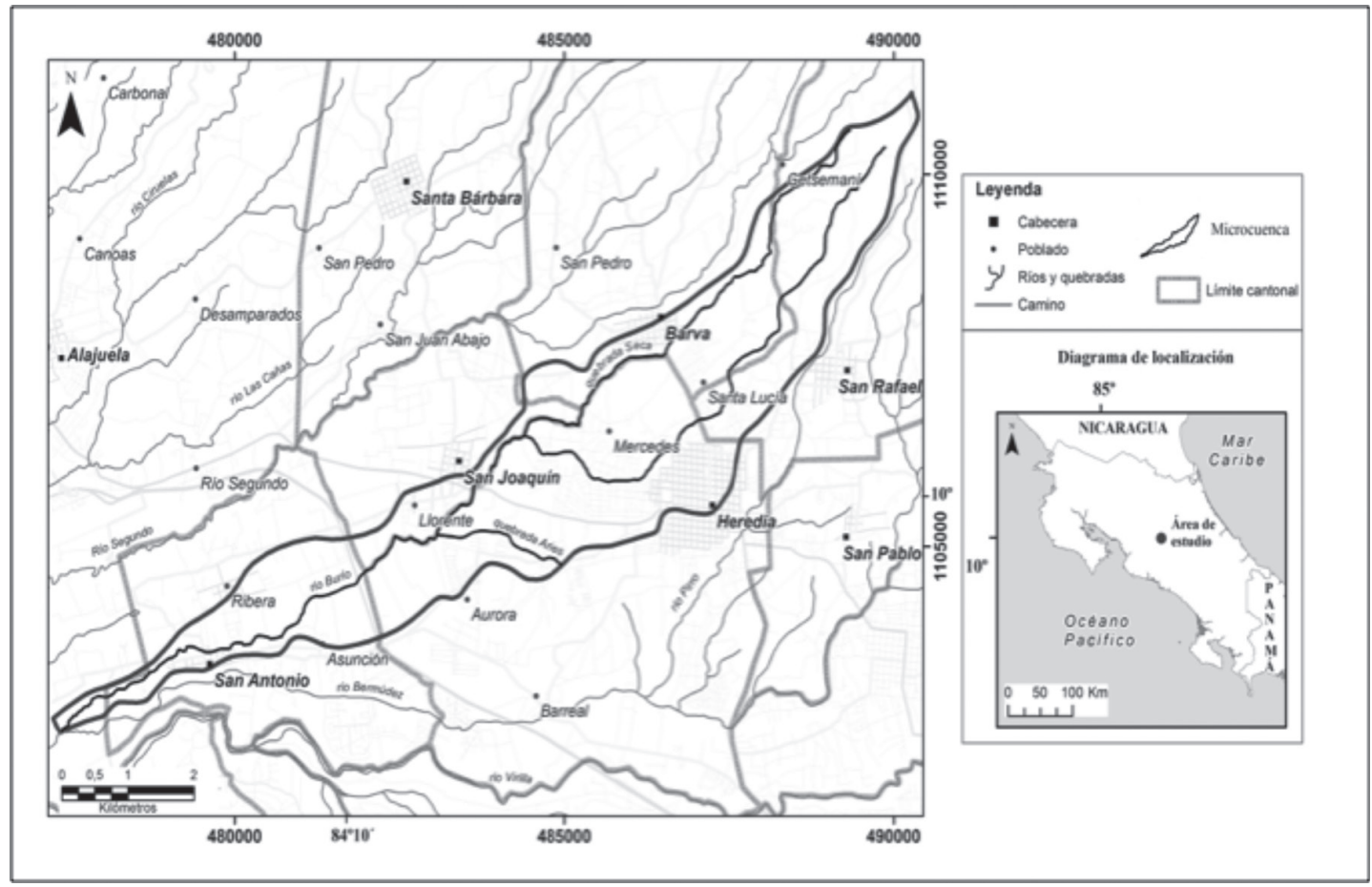

Fuente: Elaboración propia.

En los últimos 50 años, la microcuenca ha experimentado profundas transformaciones, las cuales describen la transición de usos de la tierra agrícolas a urbanos. En esta temporalidad, se manifiestan cambios en la dinámica poblacional y su estructura, al convertir la microcuenca en un espacio con alta densidad urbana.

Regionalmente, la geomorfología del área de estudio presenta dos unidades, una de origen volcánico y una subunidad designada Relleno Volcánico del Valle Central, definida por un relieve plano ondulado, modelado en parte por la actividad eruptiva de los edificios volcánicos recientes (Bergoeing y Malavassi, 1982).

El origen volcánico ha derivado materiales y suelos con una alta capacidad de infiltración natural. Estos cumplen una función muy importante para la regulación de la escorrentía superficial y recarga de acuíferos (Según Losilla, Rodríguez, Schosinsky, Stimson y Bethune, citado por
ICE $(2008,14))$. En un medio donde a lo largo del año se presentan altas precipitaciones.

La distribución de la lluvia presenta precipitaciones máximas anuales de $2.400 \mathrm{~mm}$. Según las series de registros climatológicos del IMN, en el periodo 1982 - 2007, la precipitación media anual correspondió con $2.395 \mathrm{~mm}$ en Heredia; mientras que en Santa Lucía de Barva se registraron $2.369 \mathrm{~mm}$. De igual forma, la precipitación mínima anual correspondió con $1.850 \mathrm{~mm}$ principalmente en la parte baja de la microcuenca (PRUGAM, 2009).

\section{Metodología}

Se aplicó un proceso de georeferenciación a un conjunto de fotografías aéreas para 1960, 1990 y 2005 para detectar cambios de usos de la tierra en el área de estudio. El proceso de georeferenciación es definido por Fallas (2003) como: "el proceso utilizado para relacionar la 
posición de un objeto o superficie en un plano o en archivo raster o vectorial con su posición en la superficie terrestre".

Para comprobar cuáles usos de la tierra favorecen más la impermeabilización, Moscoso y Romero (2007) analizaron los efectos del cambio del uso de la tierra sobre la escorrentía superficial en tres cuencas urbanizadas en Valparaíso, Chile, mediante indicadores como el coeficiente de escorrentía. Esta investigación determinó cómo los usos urbanos con tasas de impermeabilización más altas han reemplazado a las coberturas de bajos índices de impermeabilización, al provocar una serie de alteraciones en los componentes del ciclo hidrológico.

Martínez de Azagra y Navarro (1996) definen el término coeficiente de escorrentía como el cociente entre los valores de la escorrentía superficial y la precipitación caída. En otras palabras, es la relación entre la tasa de escorrentía y lluvia precipitada en una cuenca. La importancia de incorporar el indicador coeficiente de escorrentía radica en que es ampliamente utilizado por la ingeniería para el cálculo del caudal de diseño en alcantarillado pluvial; de ahí que esta variable incorpore elementos físicos como la cobertura del suelo, la intensidad de la lluvia y la pendiente, determinantes en la capacidad de intercepción, infiltración y escorrentía en la cuenca.

$\mathrm{Al}$ analizar tres momentos históricos en el área de estudio (1960, 1990 y 2010), se identifican cuáles coberturas y usos de la tierra (pastos, cultivos, bosque, asfalto, cemento), por su naturaleza, minimizan o incrementan la escorrentía en una cuenca. Para relacionar los tipos de coberturas de una cuenca con su capacidad para generar escorrentía durante una tormenta, se ha utilizado la matriz de coeficientes de escorrentía, con el fin de estimar caudales máximos, mediante el método racional propuesto en Chow, Maidment y Mays (1988). El cuadro 1 muestra dichos coeficientes para tormentas con diferentes periodos de retorno. El coeficiente de escorrentía oscila entre 0 y 1 , el valor depende del tipo de cobertura y la pendiente del terreno.

Es mediante el mapeo de usos de la tierra y pendientes que esta matriz permite construir escenarios geográficos de impermeabilización de suelos para diferentes temporalidades. La pendiente es un factor muy relevante en la escorrentía; pues determina la dirección y velocidad del flujo. En la matriz de Chow, Maidment y Mays (1988), se considera que a mayor pendiente, mayor coeficiente de escorrentía.

Por tal razón, se elaboró un mapa de pendientes, a partir de un modelo de elevación digital elaborado con curvas de nivel, resultantes de la cartografía del Programa de Regularización de Catastro y Registro, escala 1: 25.000. Este mapa de pendientes fue construido con la extensión 3D Analyst en el software ArcGis 9.2.

Finalmente, la siguiente etapa requiere tareas de geoprocesamiento y álgebra de mapas entre el uso de la tierra, las pendientes y los coeficientes de escorrentía correspondientes a cada cobertura. El resultado de esta operación define los escenarios de impermeabilización para los años 1960, 1990 y el 2010. 
Cuadro 1

Coeficientes de escorrentía

\begin{tabular}{ccccc}
\hline Tipo de superficie & 2 & 5 & 10 & 25 \\
& años & años & años & años \\
\hline Asfalto & 0,73 & 0,77 & 0,81 & 0,86 \\
Cemento y tejados & 0,75 & 0,8 & 0,83 & 0,88 \\
Áreas en transición & & & & \\
Pendiente (0-2\%) & 0,38 & 0,42 & 0,45 & 0,48 \\
Pendiente (2-7\%) & 0,39 & 0,43 & 0,46 & 0,49 \\
Pendiente (>7\%) & 0,4 & 0,44 & 0,47 & 0,5 \\
Zonas verdes & & & & \\
Pendiente (0-2\%) & 0,32 & 0,34 & 0,37 & 0,4 \\
Pendiente (2-7\%) & 0,37 & 0,4 & 0,43 & 0,46 \\
Pendiente (>7\%) & 0,4 & 0,43 & 0,45 & 0,49 \\
Cultivos & & & & \\
Pendiente (0-2\%) & 0,31 & 0,34 & 0,36 & 0,4 \\
Pendiente (2-7\%) & 0,35 & 0,38 & 0,41 & 0,44 \\
Pendiente (>7\%) & 0,39 & 0,42 & 0,44 & 0,48 \\
Pastizales & & & & \\
Pendiente (0-2\%) & 0,25 & 0,28 & 0,3 & 0,34 \\
Pendiente (2-7\%) & 0,33 & 0,36 & 0,38 & 0,42 \\
Pendiente (>7\%) & 0,37 & 0,4 & 0,42 & 0,46 \\
Bosques & & & & \\
Pendiente (0-2\%) & 0,22 & 0,25 & 0,28 & 0,31 \\
Pendiente (2-7\%) & 0,31 & 0,34 & 0,36 & 0,4 \\
Pendiente (>7\%) & 0,35 & 0,39 & 0,41 & 0,45 \\
\hline
\end{tabular}

\section{Resultados y discusión}

Escenario de impermeabilización 1960

Para analizar los orígenes y evolución de la impermeabilización en el área de estudio, se debe considerar que, a partir de los años cincuentas del siglo XX, Costa Rica experimentó un acelerado crecimiento poblacional y llegó a un millón de personas en 1956. Consecuentemente, hubo cambios socioespaciales en la dicotomía urbano- rural, y se disparó la densidad poblacional.

En 1950, el 58\% de la población era rural en los cantones estudiados, especialmente localizada en los cantones periféricos a Heredia; es decir, Barva, San Rafael, Flores y Belén. Para el periodo 1950-1963, la población aumentó en 22.388 personas, un $57 \%$. El cantón Heredia fue el más que creció con 11.021 habitantes, especialmente concentrado la zona urbana (Cuadro 2).

Fuente: Chow, Maidment y Mays, 1988.

Cuadro 2

Distribución de la población rural y urbana en 1950 y 1963

\begin{tabular}{cccccccccc}
\hline \multirow{2}{*}{ Cantones } & \multicolumn{3}{c}{1950} & \multicolumn{3}{c}{1963} \\
\cline { 2 - 9 } & Urbana & $\%$ & Rural & $\%$ & Urbana & $\%$ & Rural & $\%$ \\
\hline Heredia & 11.967 & 86 & 7.931 & 35 & 19.249 & 80 & 11.670 & 35 \\
Barva & 709 & 5 & 4.554 & 20 & 1.817 & 8 & 6.687 & 20 \\
Flores & 320 & 2 & 2.560 & 11 & 892 & 4 & 3.270 & 10 \\
San Rafael & 288 & 2 & 4.966 & 22 & 899 & 4 & 8.170 & 24 \\
Belén & 569 & 4 & 2.657 & 12 & 1.167 & 5 & 3.614 & 11 \\
Total & 13.853 & 100 & 22.668 & 100 & 24.024 & 100 & 33.411 & 100 \\
\hline
\end{tabular}

Fuente: Dirección General de Estadística y Censos. Censos de la población, 1950 y 1963. 
Esta situación contrastaba con el paisaje rural de la época, ya que, para 1960, el 88\% del uso de la tierra se relacionaba con actividades primarias. La naturaleza de estas coberturas agroforestales suponía que los procesos hidrológicos (intercepción, infiltración y escorrentía) estaban más regulados comparativamente con las condiciones de impermeabilización de la actualidad.

Dentro de las superficies permeables, el café era la principal cobertura agroforestal. Este cultivo predominaba al ocupar el $63 \%$ del área de estudio. A pesar de ser un cultivo introducido, este se caracteriza por ser un agrosistema que intercepta y regula de buena manera la precipitación, en relación con otros tipos de coberturas vegetales.

En general, la mayor proporción de la lluvia que ingresa a los cafetales, ya sea a libre exposición solar o bajo diferentes tipos de sombra, es retenida por la parte aérea del arbusto, con valores de intercepción del 56\%, mientras que un $38 \%$ de la precipitación que llega al suelo se infiltra y el restante $6 \%$ escurre (Giraldo y Jaramillo, 2004). En el caso del bosque nativo, este es el que mejor regula los procesos hidrológicos en una cuenca; no obstante, la cobertura forestal ha sido fuertemente sometida y presionada por las actividades socioeconómicas, ya sea para cultivar café o pastos. Reflejo de esto son los años sesenta cuando esta cobertura constituía menos del 3\% del área de estudio, y, al igual que hoy, la mayoría estaba confinada a las márgenes de las quebradas Seca, Aries y el río Burío.

En contraste con las coberturas agroforestales (Figura 2), la impermeabilización de suelos para el mismo año representaba un 9,5\% del área de estudio, aproximadamente 214 hectáreas, y correspondían con el territorio ocupado por la ciudad de Heredia y los núcleos periféricos; es decir, Barva, San Rafael y San Joaquín. Es decir, el espacio urbanizado se limitaba a las cabeceras cantonales y estas eran enlazadas por una red vial básica. La predominancia de coberturas agroforestales brindaba regulación más pausada a los procesos hidrológicos, especialmente las transiciones entre los componentes intercepción, infiltración y escorrentía. Situación que cambiaría en el corto plazo, por la explosión demográfica que se desarrollaba en Costa Rica y que repercutiría espacialmente en estos componentes por la urbanización progresiva del área de estudio. 


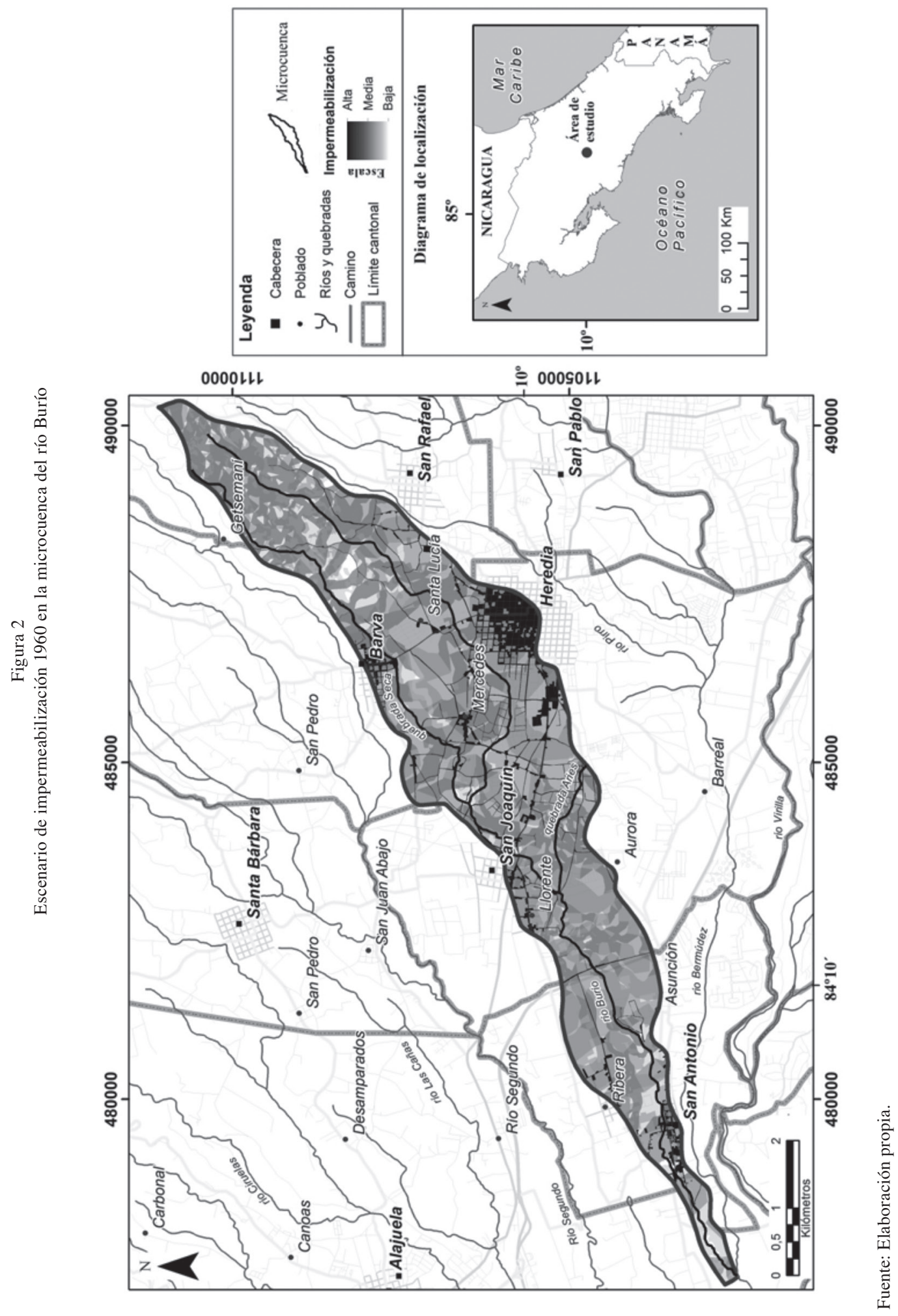




\section{Escenario de impermeabilización 1990}

El proceso de impermeabilización de suelos en la microcuenca incrementó a finales del siglo XX. Este se acentuó debido a una significativa transición poblacional rural-urbana en el área de estudio, pues la población urbana en 1963 representó un 42\% de los habitantes, en 1973 constituyó un 49\%, y en 1984 correspondía con el 51\% de la población (Cuadro 3).

Dado el crecimiento urbano en la zona, las superficies compuestas por techos y cemento aumentaron 481 ha más que en 1960. Este crecimiento de coberturas impermeables fue impulsado por la ampliación de vías principales y secundarias.

Cuadro 3

Distribución de la población rural y urbana en 1973 y 1984

\begin{tabular}{|c|c|c|c|c|c|c|c|c|}
\hline \multirow{2}{*}{ Cantones } & \multicolumn{4}{|c|}{1973} & \multicolumn{4}{|c|}{1984} \\
\hline & Urbana & $\%$ & Rural & $\%$ & Urbana & $\%$ & Rural & $\%$ \\
\hline Heredia & 19.176 & 64 & 11.927 & 35 & 41.439 & 69 & 13.457 & 23 \\
\hline Barva & 2.631 & 9 & 7.817 & 23 & 3.911 & 6 & 15.022 & 26 \\
\hline Flores & 2.677 & 9 & 2.284 & 7 & 3.327 & 5 & 5.688 & 11 \\
\hline San Rafael & 3.491 & 12 & 7.588 & 22 & 7.141 & 12 & 15.730 & 27 \\
\hline Belén & 1.888 & 6 & 4.592 & 13 & 4.559 & 8 & 7.434 & 13 \\
\hline Total & 29.863 & 100 & 34.208 & 100 & 60.377 & 100 & 57.331 & 100 \\
\hline
\end{tabular}

Fuente: Dirección General de Estadística y Censos. Censos de la población, 1973 y 1984.

Muchos accesos internos a fincas en 1960 se incorporaron como vías de comunicación entre los poblados existentes y las nuevas urbanizaciones, y facilitaron los frentes de absorción urbana entre Heredia y el resto de cantones. El crecimiento de la cobertura asfáltica paralelamente a la expansión de usos urbanos (residencial, comercial e industrial) representó un crecimiento del $28 \%$ de la red vial entre 1960 y 1990. En general en el escenario de 1990, las superficies impermeables representaron el $32 \%$ de la microcuenca.

Al comparar el escenario de impermeabilización del año 1990 con el de 1960, se puede afirmar que hubo contracción de superficies permeables, como es el caso de la actividad cafetalera, con fuerte presencia en los años sesentas, pues comprendía el 63\% del área de estudio, mientras que en 1990 decreció hasta alcanzar un $46,5 \%$ y cedió terreno al desarrollo residencial que aumentó en 368 hectáreas durante este periodo. Las superficies impermeables tales como asfaltos, cementos y tejados que existían en 1960 cubrían el 9,5\% de la microcuenca y se expandieron casi a una tercera parte del área de estudio $(32,4 \%)$ en 1990.
El decrecimiento de coberturas que cumplen un rol determinante en el proceso de intercepción, principalmente café, y el aumento de la mancha urbana, a partir de 1960, tuvo que modificar el aporte de la escorrentía a los picos de descarga cuando la microcuenca era sometida a una tormenta extraordinaria.

Mientras que en la parte alta de la microcuenca, al norte de las cabeceras de Barva y San Rafael de Heredia, la dominancia de actividades agropecuarias ofrecían condiciones de baja impermeabilización de suelos. Con el transcurrir de los años, la expansión de la ciudad de Heredia se focalizó hacia el oeste, esta situación alteró el uso tradicional de la tierra en distritos como Mercedes, donde pronto se daría paso a una transición del uso agrícola hacía el urbano, especialmente el residencial. Situación similar se dio en el sector de San Joaquín de Flores y San Antonio de Belén, impulsados por el incremento de usos residencial e industrial.

En la figura 3 se observa, como en la parte media de la microcuenca, que las superficies urbanas constituían una mancha continua entre Barva, Santa Lucía y la ciudad de Heredia. 


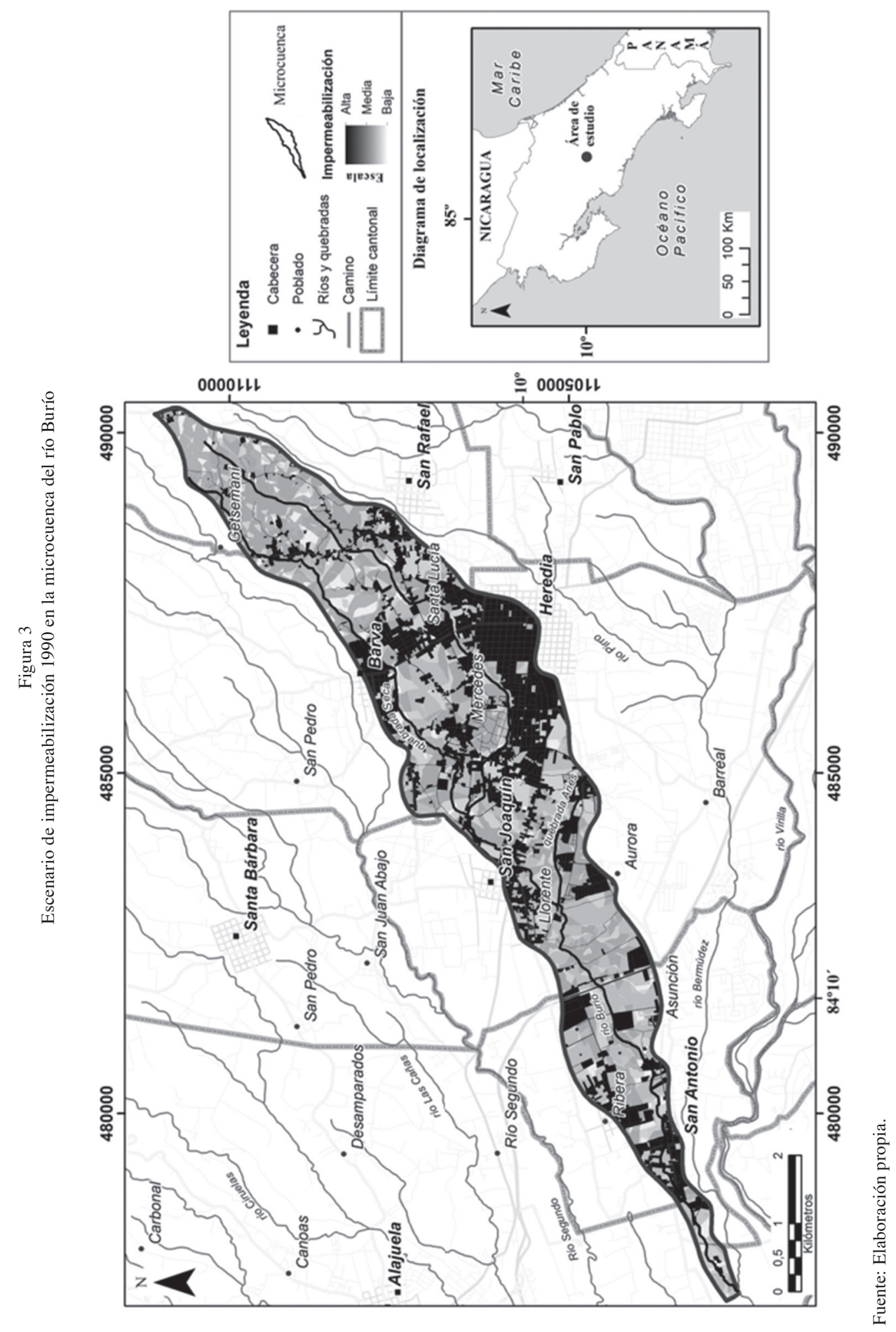




\section{Escenario de impermeabilización 2010}

En la actualidad, la microcuenca del río Burío manifiesta una intensificación del uso de la tierra, principalmente en la parte media-baja. Por ejemplo, los sectores de Asunción (Belén) y San Francisco (Heredia) dotan tierras para el desarrollo del sector industrial, bajo el sistema de zonas francas. Mientras que el distrito Mercedes (Heredia) presenta una orientación a los nuevos proyectos residenciales.

El uso actual de estos terrenos se puede explicar, debido a que el área de estudio posee condiciones muy atractivas para el desarrollo industrial, comercial y residencial. Según el Índice de Competitividad Cantonal (PROCOMER, 2009), esta zona ofrece un excelente clima de inversión empresarial, principalmente en Heredia, Flores y Belén, que parecen constituirse en un "corredor" de alta inversión, con elevados niveles de innovación e infraestructura, producto de sus exportaciones de alta tecnología.

La cartografía de uso de la tierra 2010 mostró que el uso residencial es el más importante de la microcuenca, con un 33\%. Las razones se fundamentan en el crecimiento urbano desde los centros cantonales hacia la periferia. Principalmente porque en los últimos años han proliferado numerosos proyectos de condominios y residenciales acompañados del comercio, así como complejos industriales y de servicios.

La alta conurbación se refleja en el último periodo 2000-2010, pues los distritos periféricos a la ciudad de Heredia, San Josecito, Mercedes y San Francisco, presentan una alta densidad poblacional, así como Barva, San Joaquín, San Rafael y San Antonio, todos cabeceras de municipio, cuyas densidades son media-altas.

En otros sectores de la microcuenca, el panorama seguía la misma tendencia. En Los Ángeles, San Roque y Santa Lucía el proceso ha sido más lento, debido a que se imponen las prácticas agropecuarias sobre cualquier otras. Mientras que La Rivera y Asunción han sufrido un proceso de transformación importante, marcado por la proliferación del área urbana en transición, que se espera sea utilizada para la construcción de vivienda, industria o comercio.

En el escenario actual de impermeabilización (Figura 4), se identifica que las superficies de asfalto, cemento y tejados representan el 50,8\% del área de estudio. En contraparte, las coberturas permeables, tales como: bosque, pastizal, zona verde y cultivos constituyen el $35 \%$. Actualmente, estas coberturas agroforestales están en declive respecto al crecimiento de la mancha urbana.

El café tradicionalmente tapizaba el paisaje del área de estudio. Hoy, se dedican unos 275 ha para esta actividad agrícola y unas 83 ha adicionales en otros cultivos. La mayoría de los cafetales se localizan en San Rafael, mientras que los ubicados en la parte media de la microcuenca tienden a ser espacialmente discontinuos.

Del mapa de impermeabilización se obtuvo que el $60 \%$ del área de cultivos se ubica en pendientes superiores al 7\%. El segundo grupo lo constituye la cobertura de pastizales (pasto, pasto con árboles y charral), ubicados en un $63 \%$ en pendientes superiores al $7 \%$. 


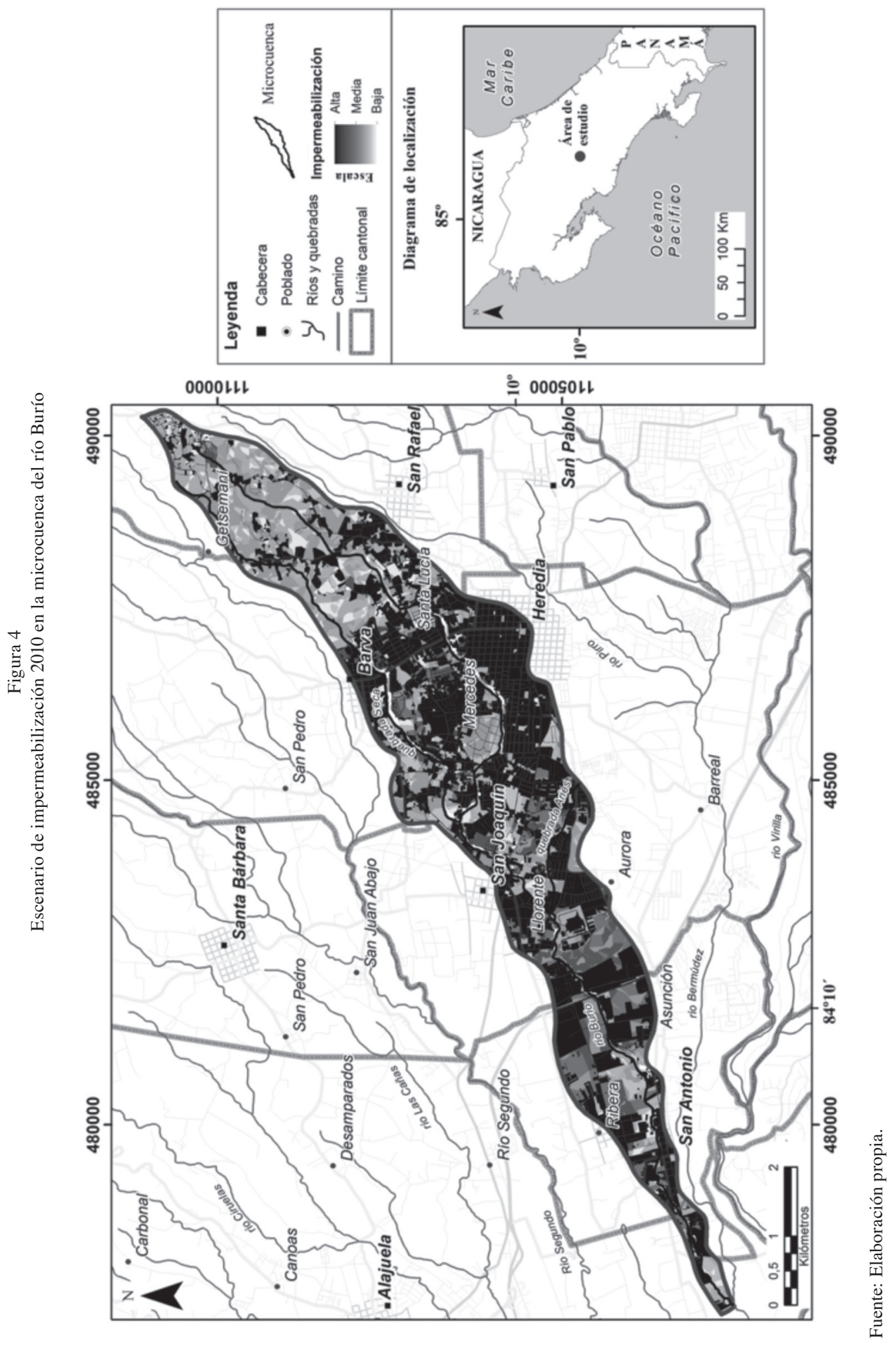


Una situación a destacar es el papel irrelevante que en la actualidad tiene la cobertura bosque, dentro del grupo de coberturas permeables; esto debido a su escasa extensión. Las manchas forestales en Barva y San Rafael se encuentran directamente asociados con la presencia de café y pasto arbolado, mientras que en las partes bajas de la microcuenca su presencia es ínfima, ya que son relictos de paisajes rurales.

Según el mapa del uso de la tierra 2010, la oferta actual de lotes potenciales para construcción de comercios, industrias o residencias es casi de 300 hectáreas. Estos terrenos se encuentran distribuidos en la sección media-baja de la microcuenca del río Burío; es decir, en distritos como Asunción, La Ribera y San Antonio (Belén), San
Francisco y Mercedes (Heredia). Esto ha volatilizado las áreas en transición, con perspectivas a urbanizarse, lo cual podría incrementar las áreas impermeabilizadas en el corto y mediano plazo.

Para prever la velocidad con que los terrenos en transición se incorporarían a la mancha urbana de la microcuenca, el tamaño es buen indicador de la probabilidad de cambios de uso, pues se esperaría que los terrenos más pequeños sean más efímeros en el mercado de compra y venta de terrenos. En este caso, los lotes menores a $500 \mathrm{~m}^{2}$ representan el $1 \%$, por lo que en un plazo de meses podrían ser dedicados a la construcción, mientras que las propiedades mayores a 1 ha agrupan el $64,5 \%$, y tendrían una transición más lenta hacia espacios urbanos (Figura 5).

Figura 5

Tamaño de las áreas urbanas en transición 2010

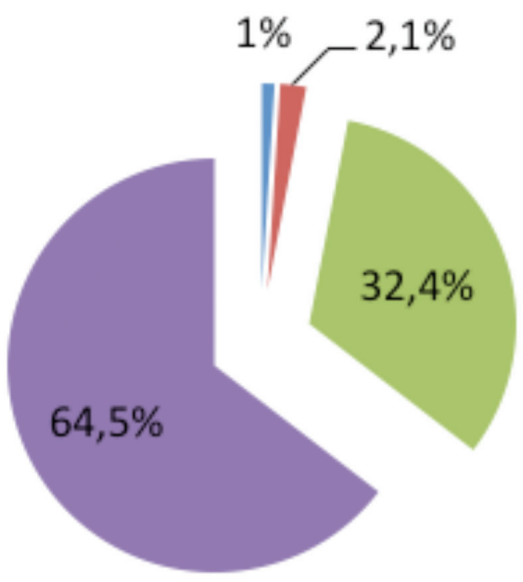

100 a $500 \mathrm{~m}^{2}$

501 a $1000 \mathrm{~m}^{2}$

1001 a $10000 \mathrm{~m}^{2}$

más de 10000 m²$^{2}$

Fuente: Elaboración propia.

La velocidad de trasformación de uso de la tierra estaría regulada por el crecimiento poblacional. Según el Censo de Población 2011, los cantones estudiados albergan una población de 251911 personas, mientras que el Centro Centroamericano de Población proyecta para el año 2030 un crecimiento de un 22,5\%, es decir 313.883 habitantes. Estas cifras repercutirán en una alta demanda de tierras, al menos para satisfacer al sector vivienda.
Como consecuencia, las áreas urbanas en transición en el corto y mediano plazo son trascendentales en la dinámica de los procesos hidrológicos futuros. La trasformación e incorporación de estos espacios a la mancha urbana y, por lo tanto, como superficies impermeables, dispararía la escorrentía y aumentaría la amenaza de crecidas con periodos de retorno cada vez más cortos. 


\section{Conclusiones}

El analizar tres escenarios de impermeabilización contrastantes en el tiempo permitió exponer cuáles coberturas por su naturaleza regulan la escorrentía o perturban los procesos hidrológicos en una microcuenca; fundamentalmente por el decrecimiento progresivo de las coberturas agroforestales, como el café, pues el bosque fue eliminado casi en su totalidad antes de la segunda mitad del siglo XX para dedicar estas tierras a la agricultura. En contraparte, el crecimiento de la mancha urbana, a partir de la explosión demográfica, tuvo un efecto acumulativo negativo en los procesos hidrológicos, principalmente en el componente escorrentía.

La expansión urbana articuló los núcleos periféricos a la ciudad de Heredia mediante un proceso de conurbación. Durante este, los caminos internos de los cafetales del área de estudio se convirtieron en nuevos enlaces que conectaban los proyectos urbanos emergentes.

Debido a la creciente impermeabilización de suelos con asfalto, cemento y techos en 1960 (9,5\%), 1990 (32\%) y 2010 (51\%), es un hecho que el aporte de la precipitación efectiva en la microcuenca, incrementó el volumen y la velocidad, al incorporar rápidamente la escorrentía a los cauces, y produjo que los picos de descarga incrementaran en magnitud y frecuencia en comparación con un área de drenaje menos intervenido.

Actualmente, el área de estudio manifiesta intensos y volátiles cambios en el uso de la tierra, pues sus tierras están muy cotizadas en el mercado de bienes raíces, fundamentado principalmente en su atractivo y en las ventajas comparativas ofrecidas por la zona al formar parte de un "corredor" de alta inversión entre Heredia y Belén, principalmente para fines comerciales e industriales.

\section{Referencias bibliográficas}

Bergoeing, J y E. Malavassi. (1982). Geomorfología del Valle Central de Costa Rica: explicación de la carta geomorfológica 1:50.000. Universidad de Costa Rica, Departamento de Geografía. San José.
Chow, V; Maidment, D y L. Mays. (1988). Applied Hydrology. Editorial McGraw-Hill. Nueva York.

Centro Centroamericano de Población (C.C.P). (2011). Datos de población para los cantones de Heredia, Alajuela, Belén, Barva, San Rafael y Flores, proyecciones para los años 2010, 2025 y 2030 (Archivo de datos). http://ccp.ucr.ac.cr/. Consultado en Marzo. 2011.

Dirección General de Estadística y Censos. 1953. Censo de la población de Costa Rica, mayo de 1950. Talleres de la Dirección General de Estadísticas y Censos. San José.

Dirección General de Estadística y Censos. 1964. Censo de la población de Costa Rica, agosto de 1963. Talleres de la Dirección General de Estadísticas y Censos. San José.

Dirección General de Estadística y Censos. 1974. Censo de la población de Costa Rica, mayo de 1973. Talleres de la Dirección General de Estadísticas y Censos. San José.

Dirección General de Estadística y Censos. 1984. Censo de la población de Costa Rica, julio de 1984. Talleres de la Dirección General de Estadísticas y Censos. San José.

Fallas, J. (2003). Proyecciones cartográficas y datum. ¿Qué son y para qué sirven? Telesig, Universidad Nacional. http://www. mapealo.com/Costaricageodigital/Documentos/alfabetizacion/proyeccion_datum. pdf. Consultado en Junio. 2011.

Giraldo, J y R. Jaramillo. (2004). Ciclo hidrológico y transporte de nutrimentos en cafetales bajo diferentes densidades de sombríos de guamo. Rev. Cenicafé 55(1): 52-68. http://www.cofenac.org/bibliografia/base. php?id=1112 Consultado en mayo del 2011.

I.C.E. (2008). Valoración del trasvase del Río Quebrada Seca. Etapa 1 del contrato "Estudio factibilidad para el transvase de la Quebrada Seca".

I.M.N. (2011). Datos de precipitación mensual de la estación Santa Lucía (111) y Aeropuerto Juan Santamaría (169) 1983-2011. http://www.imn.ac.cr/IMN/MainAdmin. aspx?_EVENTTARGET=ClimaCiud ad\&CIUDAD $=8$. Consultado en agosto. 2011. 
I.N.E.C. (2012). X Censo Nacional de Población y VI de Vivienda 2011. http://www.inec. go.cr/. Consultado en enero. 2013.

Martínez de Azagra, A y J. Navarro. 1996. Hidrología forestal. El ciclo hidrológico. Universidad de Valladolid. Valladolid. http:// www.oasification.com/archivos/Coeficientes $\% 20$ de $\% 20$ escorrent $\%$ C3\%ADa.pdf. Consultado en Octubre. 2010.

Masís, R y Vargas, H. (2011). Análisis de los cambios del uso de la tierra en 1960, 1990, 2010 y su efecto en el comportamiento hidrológico en la microcuenca del río Burío, Heredia, Costa Rica. Tesis de Licenciatura. Escuela de Geografía. Facultad de Ciencias Sociales. Universidad de Costa Rica. San José, Costa Rica.

M.I.V.A.H, M.I.N.A.E, P.N.U.M.A y Observatorio del Desarrollo. (2006). GEO Gran Área Metropolitana del Valle Central de Costa Rica: Perspectivas del Medio Ambiente Urbano (www.pnuma.org/deat1/ pdf/2006GEOGAMdeCostaRica.pdf). (Consultado en Mayo. 2011).

Moscoso, C y H. Romero. 2007. Cambios en los usos y coberturas de suelo, áreas totales impermeabilizadas y coeficientes de escorrentía en cuencas urbanizadas de Valparaíso y Viña del Mar 1980 2005. http://captura.uchile.cl/jspui/bitstream/2250/5623/1/
Moscoso_Anales.pdf. Consultado en Junio. 2010.

PROCOMER y Observatorio del Desarrollo de la Universidad de Costa Rica. (2009). Índice de Competitividad cantonal. San José.

PRUGAM. (2009). Elaboración, Actualización y Homologación de los planes Reguladores de la Gran Área Metropolitana, Cantón de Heredia: Fase I y II Análisis y Diagnósticos. (http://www.heredia.go.cr/index. php/Descargasll-documentos/Ver-documento-detailless/529-Fase-1-y-2-Analisisy-diagnostico.html). (Consultado en Mayo. 2011).

Ruberto, A; C. Depettris; J. Pilar; A. Prieto; S. Gabazza y S. Zárate. (2006). Impacto hidrológico por incremento de las áreas impermeables en cuencas urbanas subcuenca Cisterna, Resistencia, Chaco. Facultad de Ingeniería. Universidad Nordeste. Chaco. http://www.unne.edu.ar/Web/cyt/ cyt2006/07TecnologicasV2006-T-054.pdf .Consultado en Agosto, 2010.

Stanuikynas, T y D. Van. (2000). Imperious Surface Methodology. A methodology for defining and assessing impervious surfaces in the Raritan river basin. New Jersey Water Supply Autority. http://nemo.uconn. edu/tools/impervious_surfaces/pdfs/Stanuikynas_etal_2000.pdf). Consultado en Octubre. 2010. 